\title{
Pratiques
}

Linguistique, littérature, didactique

191-192 | 2021

Théâtre contemporain : Écritures et Représentations

\section{Le réel saisi par l'écriture sur les scènes contemporaines}

Entretien de Delphine Edy avec Stanislas Nordey

The real captured by writing on contemporary stages. Interview between

Delphine Edy and Stanislas Nordey

\section{Delphine Edy}

\section{(2) OpenEdition}

\section{Journals}

Édition électronique

URL : https://journals.openedition.org/pratiques/10742

DOI : 10.4000/pratiques.10742

ISSN : 2425-2042

Éditeur

Centre de recherche sur les médiations (CREM)

Référence électronique

Delphine Edy, «Le réel saisi par l'écriture sur les scènes contemporaines », Pratiques [En ligne],

191-192 | 2021, mis en ligne le 15 décembre 2021, consulté le 03 janvier 2022. URL : http:// journals.openedition.org/pratiques/10742 ; DOI : https://doi.org/10.4000/pratiques.10742

Ce document a été généré automatiquement le 3 janvier 2022.

(c) Tous droits réservés 


\title{
Le réel saisi par l'écriture sur les scènes contemporaines
}

\author{
Entretien de Delphine Edy avec Stanislas Nordey \\ The real captured by writing on contemporary stages. Interview between \\ Delphine Edy and Stanislas Nordey
}

\section{Delphine Edy}

1 Stanislas Nordey (S.N.) est à la fois un acteur reconnu et apprécié, mais aussi un grand metteur en scène, avec à son actif plus d'une centaine de mises en scène. Il est également l'actuel directeur du Théâtre national de Strasbourg (TNS) et de son école. Depuis son arrivée en 2014, il a engagé un important travail collectif, en collaboration avec une vingtaine d'artistes associés - auteurs, acteurs et metteurs en scène - à destination de publics habituellement éloignés du théâtre et dans le respect d'une parité artistiques assumée. Son grand intérêt pour les écritures contemporaines se retrouve nettement dans le projet qu'il a conçu pour le TNS.

Delphine Edy (D.E.), professeure agrégée d'allemand et docteure en littérature comparée, enseigne en classes préparatoires, en études théâtrales et en littérature comparée à l'université de Strasbourg. Spécialiste du théâtre contemporain, ses recherches portent particulièrement sur les liens entre texte et scène, la représentation $\mathrm{du}$ réel, le théâtre européen contemporain au croisement des questions d'éthique, d'esthétique et de politique.

3 Dans le cadre d'un numéro qui questionne le réel sur les scènes contemporaines, aussi bien au travers des textes que des représentations sur le plateau, le regard de cet homme de théâtre aux diverses casquettes et aux multiples combats, pour qui le théâtre est le lieu du vivre ensemble, nous a semblé central et indispensable et les échanges qui suivent en attestent. Cet entretien a eu lieu à Strasbourg, au TNS, le 5 octobre 2021.

D.E. : Vous êtes un grand défenseur des écritures contemporaines sur les plateaux de théâtre et vous êtes notamment à l'origine de la commande du texte Qui a tué mon père d'É. Louis (paru en 2018) que vous avez créé au printemps 2019 au Théâtre de la Colline. 
Ce texte pourrait être un excellent point de départ pour évoquer la question du rapport au réel, d'autant que vous avez dit vouloir monter la deuxième partie du diptyque, Combats et Métamorphoses d'une femme (paru en avril 2021). Pourquoi ? Qu'est-ce qui vous attache à cet auteur, à cette écriture ? Cela a-t-il, par exemple, à voir avec l'idée de «littérature de la confrontation » défendue par É. Louis?

5 S.N. : Je vais en effet mettre en scène Combats et Métamorphoses d'une femme. La première aura lieu au Festival des Comédiens à Montpellier en mai 2022. Par ailleurs, quand je choisis de jouer ou de monter un texte, c'est toujours la poétique qui me décide, avant le politique. Toujours. Je prends un exemple : en ce moment, je monte Ce qu'il faut dire de L. Miano et, en lisant ce texte, on pourrait se dire qu'il est politique, que c'est un texte directement ancré dans le réel, mais ce qui me fait entrer dans un texte et avoir un désir, c'est la langue. Je n'ai jamais monté un texte pour son sujet. Car je crois qu'on change le monde dans la rue et pas dans les théâtres. Peut-être que c'est vrai, bien sûr, dans les dictatures ou quand on fait des spectacles d'intervention en Afrique, sur le sida par exemple. Mais en Occident, être politique, s'il faut l'être, - et c'est un autre sujet -, c'est faire entendre aux gens de la poésie. C'est finalement le geste le plus politique qui soit me semble-t-il. Donc, pour revenir à É. Louis, j'avais lu son texte Histoire de la violence, ici au TNS en public, je l'avais invité et on est allé diner ensemble avec F. Richter et à un moment donné, je lui ai lancé, «si tu veux écrire quelque chose pour le TNS, fais-le, on sera ravis». Un an plus tard, je recevais son texte. Je n'ai pas vraiment l'habitude de fonctionner par commande, mais quand j'ai découvert son texte, c'était vraiment formidable parce qu'il y a une poétique qui me passionne et qu'il dit des choses qui me passionnent aussi. Quand j'ai monté Ciment de H. Müller [tragédie communiste en quinze tableaux mise en scène en 1995, c'est une pièce qui explore un théâtre politique où les mythes anciens resurgissent et traversent les révolutions du siècle], c'est la langue aussi qui m'a attrapé. Je suis un pasolinien, tout ce que j'ai fait au théâtre c'est une conjonction du poétique et du politique. Surtout que je n'ai jamais vraiment cru - peut-être parce que j'en ai trop vu - aux spectacles de bonnes intentions qui enfoncent des portes ouvertes, où l'on comprend que ce n'est pas bien d'être raciste et que c'est bien d'aider les autres, donc je n'y crois pas trop. En revanche, et c'est lié à mon expérience en Seine-Saint-Denis, j'ai toujours été convaincu qu'on pouvait transformer les gens par l'art. Je fais partie des gens qui croient à cela : que sa vie peut changer parce qu'on est face à un tableau, un livre ou un spectacle.

D.E. : Par ailleurs, on a beaucoup entendu ces dernières années que le théatre se voit rattrapé par le réel, que la fiction a dépassé le réel : c'était déjà le cas à Paris en 2015 avec les attentats terroristes sanglants, puis il y a eu la pandémie qui a impliqué la fermeture des théâtres, l'apparition de la distanciation sociale, le réel nous rattrape-til ? Comment analysez-vous cet état de fait, en tant qu'acteur, metteur en scène, directeur de théâtre et d'école aussi ?

7 S.N. : Ce que je vais dire va peut-être apparaître comme contradictoire avec ce que j'ai dit avant, mais je ne pense pas que le théâtre soit hors de la vie. Les spectateurs qui viennent le soir, ce ne sont pas des Superman qui se transformeraient d'un coup dans la cabine téléphonique, il n'y a pas de sas, ils viennent avec ce qu'ils ont vécu dans la journée, comme les acteurs d'ailleurs, et c'est une des choses les plus passionnantes en tant qu'acteur. Quand on arrive le soir, on arrive chargé de la journée qu'on vient de passer. On en parlait l'autre jour avec P. Rambert ; il se trouve qu'en 2015, à la fois au moment des attentats contre Charlie Hebdo et au moment de ceux du Bataclan, on était 
sur scène. Et je me rappelle la soirée du 13 novembre où on a vu toutes les petites lumières apparaitre dans la salle pendant qu'on jouait, les alertes sur les smartphones des spectateurs, et cela montre bien que le théâtre n'est pas hors du réel. C'est dans la vie, dans la ville et j'adore toujours cette anecdote qui est horrible mais qui est quand même belle, c'est celle du soldat dans le théâtre de Baltimore [racontée par Stendhal dans Racine et Shakespeare] qui tire sur Othello car il tente d'assassiner Desdémone. Il y a des spectateurs vivants dans la salle, tout peut arriver et on le sait tous, les acteurs et les spectateurs, et c'est pour cela que j'aime le théâtre. On est dans du vivant, dans du réel et donc, par définition, on est indemne de rien. Et c'est pour cela que j'ai toujours pensé que le théâtre n'était pas mis en danger par le cinéma, les réseaux sociaux... car les gens qui pratiquent le théâtre, les gens qui y vont, savent cela profondément. Là, je suis inquiet pour le cinéma, pour les salles de cinéma, parce qu'on a pu mettre en place d'autres habitudes, parce qu'on a des écrans de plus en plus grands, et peut-être que les gens vont avoir du mal à retourner au cinéma. Mais il n'y a pas de substitution au théâtre, donc ils reviendront. Après, est-ce qu'il y a des choses qui ont changé pour moi depuis 2015 ? Non. Je fais partie des gens qui pensent qu'il y a toujours des attentats partout dans le monde, et qu'on est en fait confrontés à ce moment-là à quelque chose dont on se croyait protégés. Et l'épidémie, c'est la même chose. Donc, pour moi, cela ne change pas la face du monde qui a ses soubresauts, mais, la chose quand même très particulière, c'est en effet la première fois dans l'histoire récente que tous les théâtres ont fermé. Cela m'a fait quelque chose, très symboliquement, le jour où j'ai fermé le théâtre à clé en mars 2020 et que je suis rentré chez moi.

D.E. : Ces dernières années, le théatre apparait plus politique, plus proche du réel, plus documentaire aussi. Je pense à des artistes que vous accueillez régulièrement au TNS comme J. Gosselin, T. Jolly ou C. Guiela Nguyen, mais c'est une tendance européenne, voire internationale quand on considère le travail de M. Rau, R. Icke ou C. Jatahy. Mais, parallèlement à cela, on a des plateaux de plus en plus déréalisés (au travers notamment de l'utilisation de la vidéo et des micros) et cette tendance se combine avec une remise en question de la place du texte sur le plateau. Pourtant, dans le même temps, l'écrivain metteur en scène W. Mouawad ou les metteurs en scène T. Ostermeier et É. Lacascade continuent à défendre le fait qu'il faut raconter des histoires et non les déconstruire, pour faire du sens. Comment analysez-vous ce qui apparait comme un paradoxe?

9 S.N. : Pour répondre à votre question, je vais repartir de P. Chéreau. Un jour, il a dit quelque chose qui m'a mis très en colère, et pourtant je l'aimais beaucoup: «j'abandonne le théâtre parce que j'ai besoin de me rapprocher des visages des acteurs et donc je passe au cinéma ». Je pense qu'il y a toujours eu chez les metteurs en scène de théatre un rapport ambigu de fascination et de rejet par rapport à l'image et au cinéma. Et cela passe par plein d'endroits, parfois de la jalousie, parfois de la fascination, parfois de la curiosité, etc. R. Planchon est passé au cinéma, L. Visconti n'en parlons pas. L'image est censée montrer le réel, ce qui est vrai ; ce fut le cas lors du passage de la peinture à la photographie, mais c'est paradoxal car ce n'est pas aussi simple que cela, notamment si l'on prend en compte la question du montage au cinéma. À ce sujet, j'ai vécu une expérience extraordinaire - qu'il faudrait que je raconte à des étudiants dans des écoles d'analyse de médias - lorsque j'occupais l'église SaintBernard pour défendre les sans-papiers en 1995 avec A. Mnouchkine notamment. Le matin, on rentrait rapidement chez nous avec ma compagne pour prendre une douche et un petit-déjeuner, et, un matin, on met la télévision et on tombe sur un reportage 
sur l'occupation de l'église. Il y a alors un premier plan où on voit les Africains à l'intérieur de l'église puis, immédiatement après, un deuxième plan avec un mur graffé, ce qui donne l'impression que c'est le mur de l'église. Ce montage d'images laisse donc entendre que «ces gens-là » ont écrit sur les murs des églises. Cela m'a donné une incroyable leçon sur le pouvoir de l'image, car ce n'était pas vrai. En ce moment, il y a une fascination des metteurs en scène de l'image qui passe par la vidéo, mais il faut aussi rappeler qu'il y a des raisons économiques à cette utilisation massive de la vidéo : c'est moins cher que de construire des décors, et il y a des tas de compagnie qui ont recours à la vidéo car elles peuvent avoir douze changements de décor et que ça ne coute rien alors que si elles devaient les faire construire, ce serait un gouffre financier. Mais, bien sûr, derrière cela, il y a aussi le fait que toute la génération avant la mienne, J.-P. Vincent, P. Chéreau, G. Lavaudant... étaient fascinés par faire des images ; être metteur en scène, c'est faire des images, faire des décors magnifiques. Ce qui est intéressant quand on est metteur en scène à l'opéra, c'est que la seule chose que vous demande un directeur d'opéra, c'est de faire le plus d'images possible, les plus fortes possible. Il y a donc d'abord une demande des producteurs, puis une fascination des metteurs en scène, et enfin, il y a un gout du public. Cela étant dit, concernant le théâtre documentaire - je dois dire que je vois tout, je suis un grand spectateur de théâtre -, à de très rares exceptions près, je trouve que beaucoup de ces spectacles créent du vide d'une certaine manière car, souvent, le metteur en scène ne peut en faire le tour car le sujet est trop vaste; ou alors il veut tout dire et finit par diluer le sujet et donc par ne rien dire.

10 Aujourd'hui, quand mes élèves metteurs en scène proposent un exercice (ils ont un exercice imposé et un exercice libre), ils ne proposent jamais une pièce. Jamais. Ils proposent un théâtre documentaire, soit en interrogeant des habitants pour parler de la ville, soit en assemblant des textes pour travailler par exemple sur la justice. Et il y a là pour moi un très grand danger. Il faut être très très fort. Il faut écrire comme un écrivain pour arriver à assembler des éléments qui fassent sens. Les metteurs en scène ont aujourd'hui un pouvoir démesuré dans la machine théâtrale, ils choisissent les textes, ils choisissent les acteurs, ils choisissent tout, et du coup cela peut amener à une forme d'impasse. Je me rappelle le spectacle de S. McBurney dans la cour d'honneur, il était allé jusqu'à faire écrouler le mur. Je ne me souviens pas d'un acteur, pas d'un mot prononcé, ni même du titre du spectacle. Je crois que c'est J. Copeau qui disait « l'acteur n'est pas au centre, il est le seul endroit où ça se passe ». Alors bien sûr, il y a aussi des gens comme J. Gosselin dont j'aime énormément le travail, mais c'est parce que, avant tout, ce qu'il y a au premier plan de ces spectacles, c'est la littérature et la parole.

11 Pour en revenir à la question de la narration que vous évoquiez, le cinéma raconte des histoires, il les raconte même trop bien, on n'en peut plus tellement il les raconte bien vive J.-L. Godard et A. Tarkovski quand même - car il ne fait que cela, raconter des histoires le plus clairement possible. W. Mouawad est un de mes meilleurs amis, je le connais comme si je l'avais fait, donc je connais son combat pour faire revenir les histoires, il l'a fait de manière militante - qu'il le dise publiquement ou pas - en critiquant le fait que le théatre ait abandonné les histoires, mais je ne crois pas que ce soit vrai. Je ne pense pas que le théâtre se soit tant que cela éloigné des histoires, en tout cas en France, car on a cette chance que le théâtre soit multiple : d'un côté, on a un F. Tanguy qui fait des poèmes scéniques et de l'autre, W. Mouawad qui raconte des histoires qui d'ailleurs sont parfois un peu trop racontées. Donc, je dirais de manière provocatrice qu'il ne faut pas oublier la place de la perte, l'art c'est aussi la perte, que 
ce soit en peinture ou en musique - et ce n'est pas vrai que la musique contemporaine a abandonné la mélodie, c'est juste une illusion parce que cela ressemble un peu moins bien aux mélodies faciles qu'on a l'habitude d'écouter. Toute cette histoire autour de la narration a été cristallisée au moment d'Avignon 2005 et aussi scénarisée, mise en scène, car cela arrangeait tout le monde qu'il y ait un grand combat et c'est très bien comme cela. Bien sûr, il y a des formes que l'on voit un peu plus que d'autres à un moment donné mais ce sont des histoires d'équilibre. Je pense que les formes sont toujours en mouvement - c'est d'ailleurs la force de l'histoire de l'art -, il y en a qui apparaissent plus ou moins fortement, notamment sous la pression du marché : en tant que directeur de théâtre, je sais que ce qu'on voit, c'est ce que certains veulent bien produire à un moment donné.

12 Alors, pourquoi les élèves metteurs en scène choisissent de coller, de monter des textes? J'ai par exemple donné à lire à un élève Préparatifs d'immortalité: un drame historique de P. Handke. Il le lit : il a beaucoup aimé mais pas le dernier acte. Donc, soit il coupe le dernier acte et il le réécrit, soit il ne monte pas du tout la pièce et il fait autre chose. Alors que moi j'ai toujours trouvé passionnant de monter TOUT le texte. Je me rappelle très bien quand j'ai monté Bêtes de style de P. P. Pasolini, B. Dort, une éminence à l'époque, vient voir le spectacle et me dit : «C'est bien ton travail mais la pièce est un peu bavarde, Pasolini se répète, tu aurais dû couper ça, ça ça et ça ». Et moi, du haut de mes vingt-cinq ans - j'étais un peu orgueilleux - je lui rétorque : «Une œuvre d'art est belle par ses forces et ses fragilités ». Et c'est là que le privilège du metteur en scène, est dangereux, car il y a un risque d'abus de pouvoir. Un élève metteur en scène a eu envie une fois de faire quelque chose sur le Living Theatre; je lui demande alors ce qui l'intéresse dans ce théâtre: la naissance de leur aventure, ce qu'ils ont fait dans les favelas au Brésil ? Mais il ne savait pas. Il voulait faire un truc sur le Living Theatre. C'est surement lié au fait que pour chaque génération il y a des marqueurs. Je le vois dans les dossiers lorsqu'on recrute pour l'école. Dans les dix dernières années, tous les jeunes gens ont vu Les Chiens de Navarre (compagnie créée par J.-C. Meurisse en 2005) et veulent faire quelque chose qui parte de ces formes-là. Comme, à une époque, il y a eu A. Mnouchkine. Donc, il y a des identifications très fortes à des formes qui vous sautent au visage et vous avez alors envie de vous accrocher au wagon et de prendre la suite.

D.E. : Prenons peut-être un contre-exemple pour essayer de préciser encore les choses en ce qui concerne l'écriture de plateau. Vous avez collaboré avec F. Richter dans My secret Garden ou dans Je suis Fassbinder. Son écriture entremêle parties documentaires, éléments autobiographiques et fiction, les comédiens portent leur porte nom et parlent leur propre langue, comme dans I am Europe. Et il publie ces pièces, qui sont par ailleurs traduites pour la plupart chez L'Arche.

S.N. : Oui, mais c'est parce que lui, c'est avant tout un écrivain. Donc F. Richter écrit des textes, et, son originalité, c'est qu'il procède par montage ; d'ailleurs il intègre parfois dans un spectacle un texte qu'il avait écrit pour un autre spectacle. Il part de l'écriture quand il crée un spectacle. Pour moi, les écritures de plateau, à de rares exceptions près, sont une nouvelle prise de pouvoir du metteur en scène sur l'acteur d'abord, parce que, quand on est en répétition d'une écriture de plateau, le despote, c'est le metteur en scène. L'acteur a de moins en moins de place à cet endroit-là. Quand on monte une œuvre littéraire, quelle qu'elle soit, il y a quelque chose qui se raconte, il y a une trame, une fable, cela dit quelque chose avec lequel on est d'accord ou pas. Avec P. Rambert par exemple, on ne peut pas modifier une virgule. C'est un écrivain avant 
toute chose. D'ailleurs, que ce soit pour Les Sceurs, Clôture de l'amour ou Répétition, il a écrit la pièce puis il nous a mis dans un dispositif dans lequel on était complètement libres. Je me rappelle très bien, pour Clôture de l'amour, il nous a dit, « au début c'est une diagonale, puis vous vous croisez au milieu et vous changez la diagonale et pour le reste, vous vous débrouillez ». Dans Répétition, que j'ai adoré faire, c'était encore plus radical. Il nous avait donné le début du spectacle - on entre et on se place à un endroit défini -, la fin du spectacle, et au milieu, on était entièrement libre tous les soirs ; bien sûr, on devait dire le texte dans l'ordre mais physiquement, on pouvait faire ce qu'on voulait et il avait créé un dispositif avec le créateur son et lumières qui faisait que la lumière et le son changeaient aussi tous les soirs. Cela étant, il y avait un cadre extrêmement précis qui était le texte et donc une rythmique, cela ne devait pas durer plus de deux heures et quarante minutes. Pour moi, c'est passionnant de travailler avec des gens comme P. Rambert ou F. Richter, parce qu'on est vraiment chez des écrivains : ils ont un propos, la pièce démarre là et se termine par là et on ne transige pas avec ça.

D.E. : Un théâtre « au cœur du réel » pour reprendre le titre de l'ouvrage d'É. Lacascade, cela implique de questionner les notions d'authenticité, de fidélité, de fabrication, on l'a bien vu à l'instant en parlant des images et de l'écriture de plateau, mais aussi celles de trahison et de vérité dans leur rapport au réel. L'écriture contemporaine a cette force spécifique qui fait qu'elle surgit dans le réel, dans notre réel, mais quand on choisit de mettre au plateau un texte plus ancien, un texte du répertoire, comment les choses peuvent-elles faire sens?

S.N. : Cela ne fait pas sens. Je ne vois pas pourquoi on continue à monter Tartuffe, sinon comme un témoignage d'une écriture ancienne. J'adore A. Mnouchkine, mais quand elle avait monté Tartuffe qui se passait chez les intégristes, ce n'était pas vrai, ça n'avait rien à voir, ce n'était pas le même contexte historique. Simplement, ça amuse les gens de se dire que Molière avait déjà vu tout ça, mais ce n'est pas vrai. Ce qui parle d'aujourd'hui, c'est aujourd'hui. Pour moi, c'est une paresse et une facilité d'aller chercher dans le répertoire. Je ne parle pas de la valeur littéraire bien évidemment. C'est-à-dire qu'on peut monter J. Racine parce qu'on est dingue de sa langue, mais monter Bérénice pour dire que c'est ce qui se passe à Gaza, non. Je trouve cela bête, facile et malhonnête parce que ce n'est pas vrai et qu'au moment, par exemple, où W. Shakespeare écrit, il écrit dans un contexte très particulier, dans un rapport particulier au Prince, ce dont on est libéré aujourd'hui. Donc je suis assez virulent là-dessus et je l'ai été de manière militante pour deux raisons : la relecture des classiques - l'époque de P. Chéreau, J.P. Vincent... dont on a parlé plus haut -, c'était finalement une manière de mettre le metteur en scène en avant, c'est-à-dire, « tiens, comment Chéreau a-t-il donc monté Le Misanthrope, je suis curieux de savoir ", mais on n'écoute plus Le Misanthrope. On va regarder une variation esthétique sur un thème d'une certaine manière, et cela, à un moment donné, je trouve que cela éloigne du cœur des choses qui, pour moi, est quand même l'écriture. Et le pire, c'est en général à l'opéra. Et ce n'est pas un discours réactionnaire, c'est un constat. Puisqu'on n'arrête pas de monter La Traviata, car c'est ce que les gens veulent voir, il faut bien demander aux metteurs en scène d'avoir la version la plus dingue pour qu'elle soit différente de celles qu'on a vues auparavant, mais pendant ce temps-là, on n'écoute plus la musique. On regarde comment K. Warlikowski a dynamité La Traviata.

17 Pour en revenir au réel, il est tout le temps dans le théâtre, dans l'écriture. Quand P. Claudel écrit Partage de midi ou Le Soulier de satin, le réel est au cœur de son écriture : 
il raconte sa vie avec une femme qui s'appelle Rosalie et la manière dont il en bave ; on parle beaucoup aujourd'hui d'autofiction, mais tous les auteurs font de l'autofiction, ils racontent ce qui les habitent intimement, donc la question du réel est toujours au cœur de l'œuvre.

D.E. : On peut dire cela, mais il y a quand même un contrat avec le lecteur ou avec le spectateur. Je pense à quelqu'un qui n'écrit pas directement pour le théâtre mais qui a beaucoup été mise en scène, A. Ernaux. Elle ne transige pas avec l'idée que tout ce qu'elle écrit est de l'ordre de la vérité et elle ne peut pas faire entrer un personnage fictionnel ou même donner une couleur a une personne réelle. Comme elle l'a rappelé lors d'un colloque récent [02.10.2021] organisé par la Bibliothèque nationale de France, si elle n'a toujours pas trouvé de terme exact pour nommer ce qu'elle écrit, elle reste ferme sur une chose qu'elle appelle « le pacte de vérité ».

S.N. : C'est vrai, mais, au théâtre, arrive le metteur en scène qui est un filou. Car il lui arrive souvent de faire dire à l'œuvre ce qu'il a envie qu'elle dise. Il est quand même un médiateur, un médiateur qui produit des images qui sont d'une sacrée force. Pour ma part, je suis venu au théâtre après avoir lu L'Espace vide de P. Brook, je fais partie de cette génération-là, et déjà à l'époque, je me méfiais des images. Et aujourd'hui encore, je me bats quand je mets en scène une pièce. Pendant très longtemps, j'ai refusé qu'il y ait la moindre projection vidéo dans mes spectacles, j'y suis venu très prudemment à une ou deux reprises, parce que, ce qui me fait peur dans l'image, c'est la fascination qu'on a pour elles. Si on me met un écran et un acteur sur le plateau, je ne regarde pas une seconde l'acteur, je regarde l'écran. Et le travail que J. Gosselin fournit ici est un bon contre-exemple car il n'abandonne ni le sens, ni les acteurs. Car ce sont des " gueules » d'acteurs qui apparaissent sur l'écran.

D.E. : C'est vrai, mais en même temps, encore dans son dernier spectacle Le Passé, on a du mal à résister à l'image, et on se laisse facilement dériver, en tout cas c'est l'expérience que j'ai faite en tant que spectatrice.

21 S.N. : De toute façon, on n'a pas le choix puisqu'il n'y a pas d'échappatoire, mais, pour le coup, c'est au cœur de sa recherche et je la trouve intéressante.

D.E. : Il y a encore une question que j'aimerais vous poser : avez-vous déjà eu envie d'écrire du théâtre ? Vous défendez les auteurs dramatiques, vous avez déjà dit que vous voudriez qu'ils puissent aussi diriger des théâtres, vous avez souvent rappelé que l'auteur est supérieur au metteur en scène, et que c'est une des raisons pour lesquelles vous ne coupez pas les textes... Mais qu'en est-il de vous?

S.N. : Non, je n'ai pas envie d'écrire de théâtre et je vais vous dire pourquoi. Il y a deux raisons. Une raison intime d'abord. Je suis quelqu'un d'assez pudique et, pour moi, il y a une énorme différence entre lire un roman et lire une pièce de théâtre. Quand on lit un roman, par exemple Voyage dans l'Est de C. Angot, je suis seul face au livre, vous êtes seule aussi : le roman on est "seul face ». La pièce de théâtre, il y a cinq cents personnes qui l'écoutent en même temps et surtout, autour de la table de répétition, il y a quarante personnes qui discutent pour savoir pourquoi l'auteur a écrit cela, à quoi cela correspond dans sa vie... Je trouve cela terriblement impudique d'écrire une pièce de théâtre. Car, pour être de l'autre côté, je passe mon temps à me poser toutes ces questions. La deuxième, c'est que, quand je me suis mis à lire du théâtre, il y avait déjà tellement de belles choses que je me suis dit « mais pourquoi aller perdre mon temps à écrire du théâtre ». Quand j'ai commencé à faire du théâtre, je l'ai fait comme le petit garçon que j'étais qui lisait beaucoup - des bandes dessinées ou un Fantomette - et qui 
allait donner ses livres à d'autres enfants en leur disant «tu devrais lire ça »; j'ai toujours été mu par le fait de partager mes lectures, donc, pour moi, mettre en scène, c'est faire connaitre à un grand nombre. Ma dernière découverte, c'est A. Carson, Autobiographie du rouge, et je me réjouis à l'idée de la faire connaitre à d'autres. Une des choses dont je suis le plus fier en tant que metteur en scène, c'est d'avoir fait découvrir P. P. Pasolini en France ou J.-L. Lagarce. Et enfin, pour écrire il faut du temps et je n'en ai pas, et si je trouve un jour le temps d'écrire plus sérieusement, j'écrirai tout sauf du théâtre.

D.E.: Vous venez de citer A.Carson, et je me demandais, quels sont les textes contemporains que vous auriez envie de monter aujourd'hui ou de voir sur scène parce qu'ils feraient plus sens que d'autres? Est-ce qu'il y a des urgences?

S.N. : Il y a différents types d'urgence, il y a des urgences viscérales; par exemple quand j'ai lu Les Portes de Thèbes de M. Riboulet - un texte sublime sur les enfants du Bataclan, tous, les tueurs et les victimes - c'est la poétique qui m'a accroché et j'ai immédiatement eu envie de le passer sur le plateau ; là, il pourrait y avoir une urgence. Qui a tué mon père, quand il est arrivé, je me suis dit « il faut le faire tout de suite »; et, au moment des attentats, j'ai eu envie de faire entendre de la manière la moins didactique possible que la question du terrorisme était plus complexe que la manière dont on en parlait, que pendant la guerre de 1939-1940, les nazis appelaient les résistants des terroristes, et j'ai choisi de monter Les Justes d'A. Camus. Et puis, il y a des textes qui vous accompagnent au plus long cours. A. Carson, c'est une urgence de gourmandise : j'ai envie de m'y attaquer parce que c'est sublime. Mais je pense à un autre exemple : il y a quinze ans, F. Melquiot avait écrit une pièce, Tasmanie, qui raconte le triangle entre N. Sarkozy, C. Sarkozy et J. Martin, son premier mari. C'est un texte d'une extrême violence contre N. Sarkozy qui n'est jamais nommé, ce sont des noms de fiction, et j'ai eu envie de monter ce texte tout de suite, mais aucun producteur n'a voulu monter cette pièce. C'est assez intéressant d'ailleurs et, un jour, il faudrait faire une histoire des textes qui n'ont jamais été montés parce qu'ils n'ont pas trouvé de producteurs. Et clairement, dans ce cas précis, même si cela n’a jamais été dit, c'était par peur des représailles. À cette époque, j'avais ressenti une urgence, même si le projet n'a pas abouti. Après, j'ai aussi une particularité par rapport à d'autres collègues. En général, les metteurs en scène ne lisent pas tant que cela, particulièrement du contemporain. Dans le théâtre qui parait aujourd'hui, je lis tout et cela permet qu'il y ait toujours les éblouissements auxquels on ne s'attendait pas. Je m'étais dit qu'un jour j'aurais monté tout ce que j'avais à monter - à ce jour, j'ai déjà monté plus d'une centaine de spectacles - mais en fait, quand on s'intéresse à la création contemporaine, c'est inépuisable, cela ne s'arrête jamais.

D.E. : C'est plutôt une bonne nouvelle en fait! [Rires] Par ailleurs, j'ai un étudiant de théâtre en L3 qui prépare un dossier de recherche sur la programmation de la saison 2021-2022 à Strasbourg dans les différents théâtres et il s'est rendu compte que la nature est visiblement au cœur des préoccupations, sauf au TNS. Est-ce que vous confirmez? Et si oui, comment vous l'expliquez-vous?

S.N. : C'est drôle ce que vous dites car l'année dernière j'ai passé commande à six autrices sur cette question-là précisément : A. Hilling, M. Ndiaye, L. Miano, A. Liddell, E. Jelinek et A.Carson, parce que, justement, je me disais qu'il fallait aussi que ces questions-là arrivent sur les plateaux et qu'il y avait certains spectacles d'écriture de plateau qui les traitaient, mais qu'il était important qu'il y ait des textes au répertoire. 
C'est d'ailleurs la même question sur tous les débats aujourd'hui comme l'identité binaire, transgenre, cisgenre... il y a peu de littérature - C. Pellet vient d'ailleurs d'écrire deux très beaux textes sur ces sujets, Aphrodisiaque et Les Rêveurs - et je pense que c'est toute l'importance du contemporain : féminiser les plateaux de théâtre, ce n'est pas faire jouer des rôles d'hommes à des actrices, c'est écrire des nouveaux textes, des textes d'aujourd'hui pour des actrices.

D.E. : Donc, cette question de la nature arrivera au TNS de manière décalée, et cela est lié au temps d'écriture des textes, on va donc les attendre patiemment. Une dernière question : en janvier 2021, P. Chevilley signe un article intitulé, "Stanislas Nordey, un artiste de combat». En effet, à la tête du TNS, vous multipliez les créations maison, vous promouvez la formation, mais aussi la parité homme-femme et la diversité, notamment avec le programme "Premier Acte». En 2018, vous avez également lancé un vaste chantier de réflexion sur l'avenir du théâtre avec la question « que sera le TNS dans 50 ans ? » Et aujourd'hui, quelles sont vos priorités ? Quel est votre combat?

S.N. : Il y en a un que je n'aurai pas eu le temps de mener, que j'aurai juste eu le temps d'amorcer, c'est la question du handicap. C'est pour moi une question très importante. Souvent, on voit surgir les questions à des moments inattendus car il y a des impensés. La question de la diversité en est un. Je ne pense pas vraiment qu'il y avait du racisme mais la diversité n'était pas pensée, la question n'existait pas. C'est dans l'expérience «Premier Acte » que la question du handicap est apparue. En France, on n'a pas le droit de faire de discrimination positive, et moi, je voulais qu'il y ait plus d'acteurs issus de l'immigration récente qui soient sur les plateaux et qui ressemblent à la France telle qu'elle est, mais ce n'était pas possible de le formuler ainsi. Donc, on a écrit une lettre qui disait en gros «si vous estimez être victimes de discrimination dans la pratique de votre art, vous pouvez postuler pour participer à ce programme ». La première année, sur une quarantaine de candidats, on a eu environ trente-cinq personnes venant des couches d'immigration récentes, sahariennes, subsahariennes, etc., deux personnes en surpoids et un jeune homosexuel. Et puis, petit à petit, on a eu des gens avec des handicaps, une personne avec un seul bras, un non-voyant, puis des handicaps parfois de plus en plus lourds, je pense à une jeune fille qui ne pouvait bouger qu'un doigt ou une paupière. Là, tout t'éclate au visage : comme je n'ai pas autour de moi de gens avec des handicaps lourds, c'était resté un impensé. Cette année-là, il y avait aussi un jeune homme qui a passé l'audition avec un handicap lui causant des tremblements et parfois des difficultés pour parler, on l'a pris dans l'école du TNS: c'est un comédien exceptionnel, un être exceptionnel et tout se passe très bien. Aujourd'hui, quand on parle de handicap au théâtre, c'est, d'une part, des normes européennes qu'il faut respecter, d'autre part, du rapport aux salariés - ça peut nous arranger d'engager des gens avec des handicaps car on obtient des primes, mais il y a aussi les salariés avec des handicaps légers qui ne déclarent rien car ils ont honte et ne veulent pas se voir fermer de portes - et enfin, c'est la question de la visibilité, c'est-à-dire que si aujourd'hui tu as douze ans, que tu es en fauteuil et que tu veux devenir actrice, tu ne te poses même pas la question « est-ce que cela pourrait être possible? ", car ça n'est pas possible. Il y a un beau combat à mener car il n'y a aucune raison que cela ne devienne pas possible. Absolument aucune raison. Pour moi, ces prises de conscience sont récentes, encore une fois par l'expérience de ces auditions de "Premier acte ». Donc, si j'ai un dernier combat à mener, ce serait celui-là. C'était l'un des enjeux de mon deuxième mandat mais les confinements successifs ont torpillé tout cela. Il y a des endroits où on a 
tellement de retard, qu'il faut du volontarisme, sinon les choses n'avancent pas, il faut de l'activisme. Ce n'est qu'à ce prix qu'on fera bouger la société.

\section{AUTEUR}

\section{DELPHINE EDY}

Université de Strasbourg, Accra, F-67081 Strasbourg, FranceSorbonne Université, CRLC, F-75006 Paris, France 